\title{
Space-Time Trellis Coded 8PSK Schemes for Rapid Rayleigh Fading Channels
}

\author{
Salam A. Zummo \\ Electrical Engineering and Computer Science Department, University of Michigan, Ann Arbor, MI 48109-2122, USA \\ Email: sazummo@umich.edu \\ Saud A. Al-Semari \\ Electrical Engineering Department, King Fahd University of Petroleum and Minerals, Dhahran 31261, Saudi Arabia \\ Email: semari@kfupm.edu.sa
}

Received 29 May 2001 and in revised form 17 January 2002

\begin{abstract}
This paper presents the design of 8PSK space-time (ST) trellis codes suitable for rapid fading channels. The proposed codes utilize the design criteria of ST codes over rapid fading channels. Two different approaches have been used. The first approach maximizes the symbol-wise Hamming distance (HD) between signals leaving from or entering to the same encoder's state. In the second approach, set partitioning based on maximizing the sum of squared Euclidean distances (SSED) between the ST signals is performed; then, the branch-wise HD is maximized. The proposed codes were simulated over independent and correlated Rayleigh fading channels. Coding gains up to $4 \mathrm{~dB}$ have been observed over other ST trellis codes of the same complexity.
\end{abstract}

Keywords and phrases: diversity, multiple transmit antennas, space-time codes, Rayleigh fading channels.

\section{INTRODUCTION}

As more wireless communication systems are emerging, higher data rates with improved quality of service are required. In general, diversity and error control codes are known to improve the link quality in wireless communications $[1,2]$. In particular, transmit diversity can be used to increase the transmission rate. Also, the rate can be increased using higher order signal constellations, such as MPSK and M-QAM. The conventional transmit diversity system can be viewed as a repetition code which consumes higher bandwidth [2]. Therefore, it is expected that substantial performance improvement can be achieved using more sophisticated codes, utilizing both space and time. Systems combining transmit diversity and trellis codes with high order constellations are promising to provide higher transmission rates at very good quality via providing diversity in time and space.

The concept of space-time (ST) codes had appeared first in [3], referred to as the delay diversity system, where different symbols are simultaneously transmitted via different transmit antennas. Later, the performance criteria of ST codes over quasi-static and rapid fading channels were derived in [4], where ST codes were designed for quasi-static fading channels. In $[5,6,7]$, the ST concept was applied to enhance the quality of transmission at the same bit rate of systems using single transmit antenna. Hence, the same error probability can be achieved at a lower signal-to-noise ratio
(SNR). Improved ST codes for trellis-coded modulation have been presented in [8]. Moreover, optimum ST trellis codes for quasi-static Rayleigh channels have been obtained using a new approach [9]. The basic concept of ST codes was also extended to turbo codes $[10,11]$. ST trellis codes for rapid fading channels using QPSK and 16-QAM signal constellations were presented in [12] and [13], respectively.

In this paper, two ST coded 8PSK schemes suitable for rapid fading channels are proposed. The paper starts with a general description of the ST system model. Then, the proposed codes are presented. Performance comparisons are shown in Section 4. Finally, conclusions out of this work are outlined.

\section{SYSTEM MODEL}

A typical system that employs ST coding consists of a trellis encoder, a vector block interleaver, $N$ modulators, $N$ transmit antennas, $M$ receive antennas, demodulators and a combiner, a deinterleaver, and finally a ST decoder. The block diagram of a typical ST coded system is shown in Figure 1. Throughout this work, $N$ has been set to 2 . The encoded ST signals are interleaved using a vector block interleaver. Each element in the interleaver is a vector containing $N$ symbols which are transmitted via the $N$ transmit antennas. The interleaver is used in order to break the memory of the channel so that it approaches the behavior of independent fading 


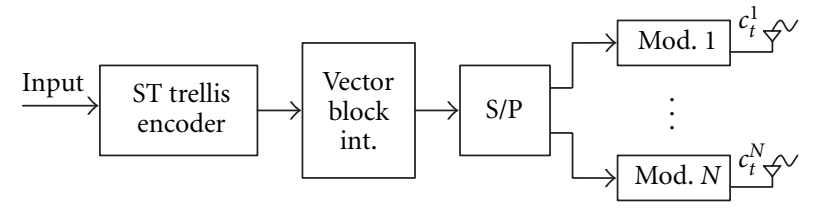

(a) Encoder.

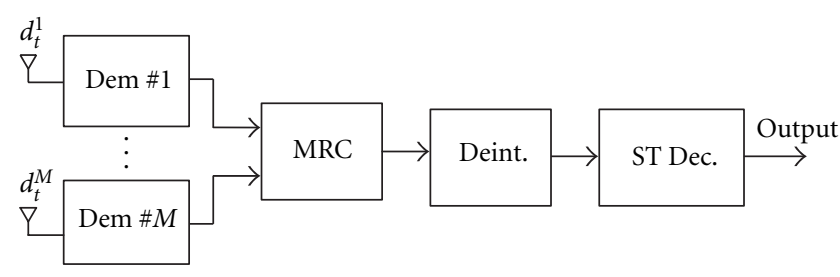

(b) Decoder.

FIgure 1: General ST system (a) Encoder, (b) Decoder.

channels, and hence the diversity provided by the coded system is fully utilized. The depth and span of the interleaver depend on the channel's fading rate $\left(f_{D} T\right)$ and the encoder's constraint length, respectively. The received signal at the $j$ th receive antenna is a noisy superposition of all transmitted symbols over all transmit antennas and is modeled as

$$
d_{t}^{j}=\sum_{i=1}^{N} \alpha_{i j, t} c_{t}^{i}+\eta_{t}^{j},
$$

where the coefficient $\alpha_{i j, t}$ is the path gain from the $i$ th transmit antenna to the $j$ th receive antenna at time instance $t$. It is modeled as independent samples of a zero-mean complex Gaussian random process with variance of $1 / N$. The $c_{t}^{i}$ is the transmitted symbol from the $i$ th transmit antenna, and $\eta_{t}^{j}$ is an additive white Gaussian noise (AWGN) sample with variance $N_{0} / 2$ per dimension. The pairwise error probability of ST coded systems over rapid Rayleigh fading channels is upper bounded as [4]

$$
P\left(\mathbf{C}_{l}, \hat{\mathbf{C}}_{l}\right) \leq \prod_{t \in \eta}\left[1+\sum_{i=1}^{N}\left|c_{t}^{i}-\hat{c}_{t}^{i}\right|^{2}\left(\frac{E s}{4 N_{0}}\right)\right]^{-M},
$$

where $\eta=\left\{t: \underline{c}_{t} \neq \underline{\hat{c}}_{t}\right\}$, and $\underline{c}_{t}=\left(c_{t}^{1} c_{t}^{2} \cdots c_{t}^{N}\right)$ is the codeword of symbols transmitted simultaneously over all transmit antennas at time $t$. The parameter $L$ defined to be the length of the shortest error event path, is referred to as the spacetime minimum time diversity (ST-MTD) of the ST code [12]. It can be visualized as the "branch-wise" Hamming distance (HD) in conventional trellis codes, by considering the whole codeword $\underline{c}_{t}$ as one symbol. The design rules of good ST codes over rapid fading channels state that for the system to achieve a diversity of $v M$, the code should have $L$ being at least equal to $v[4]$. Also, the code's ST minimum squared product distance should be maximized. It is defined over the shortest error path as

$$
\begin{aligned}
d_{P}^{2}(L)=\min & \left\{\prod_{t=1}^{L} \sum_{i=1}^{N}\left|c_{t}^{i}-\hat{c}_{t}^{i}\right|^{2} ;\right. \\
& \text { for all error path of effective length }=L\},
\end{aligned}
$$

where the sum of squared euclidean distances (SSED) is defined as the summation term in (3). The asymptotic gain is a popular parameter to compare different coding schemes. In [14], it was derived for conventional trellis codes. It is defined as the difference in the SNR values required in both coded systems to provide the same error probability. In this paper, a similar approach is used to derive the asymptotic gain of ST codes. From (2) and (3), the asymptotic coding gain of any ST coded scheme over another one can be expressed by

$$
g_{\infty}=\frac{10}{L} \log _{10}\left(\frac{d_{P}^{2}(L)_{2}}{d_{P}^{2}(L)_{1}}\right)\left(\frac{\alpha_{1}}{\alpha_{2}}\right)^{1 / L},
$$

where $\alpha_{1}$ and $\alpha_{2}$ are the multiplicities of the shortest error events in the first and second codes, respectively. This expression will be used to calculate the gain of the proposed ST codes over the available ST codes in the literature. In the following, a detailed discussion on the design of the proposed ST codes is presented.

\section{THE PROPOSED CODES}

All 8PSK ST schemes presented in this paper provide a throughput of $3 \mathrm{bits} / \mathrm{s} / \mathrm{Hz}$. The ST coded $8 \mathrm{PSK}$ scheme designed in [4], referred to as 8PSK1 here, uses a rate- $3 / 6$ trellis encoder to encode the incoming 3 bits onto 6 output bits. The 6 bits at the output of the encoder are mapped onto two 8PSK signals and transmitted over two transmit antennas. It is optimized for quasi-static fading channels, but it is presented here as a baseline for the new codes. Both the ST minimum time diversity and minimum squared product distance of the code are 2, where the shortest error event has a multiplicity of 2 .

The second 8PSK code is referred to as 8PSK2. It also uses a rate- $3 / 6$ trellis encoder. The design approach of this code maximizes the symbol-wise HD of branch labels leaving from or entering to the same state. This HD is the controlling factor in the design of multidimensional trellis codes over fading channels. In [15], the general approach for partitioning multidimensional MPSK signal space for fading channels was presented. Hence, the 4D signal space at the output of the ST encoder is partitioned using this approach. The resultant code is expected to have a higher ST minimum squared product distance than the 8PSK1 scheme. In $[12,13]$, a similar approach was used to design ST codes for rapid fading channels using QPSK and 16-QAM signal constellations, where substantial coding gains have been observed. The set partitioning of multidimensional MPSK signal space for fading channels developed in [15] is briefly reviewed in the following. 
Let $\Omega^{0}$ be the $4 \mathrm{D}$ MPSK signal set to be partitioned. In general, $\Omega^{p}$ refers to the subset at the $p$ th partitioning level. The set $\Omega^{p}$ contains $2^{6-p}$ signal points, where the number of bits at the encoder's output is 6 in this case. The SSED between signals in $\Omega^{1}$ is $2 \delta_{0}^{2}=2 \times 0.586=1.17$, where the symbol-wise HD is 1 . If the vector $\mathbf{y}_{i}=\left[\begin{array}{lll}y_{i}^{2} & y_{i}^{1} & y_{i}^{0}\end{array}\right]$ is the bit representation of $i$ th codeword at the output of the encoder, then the vector $\mathbf{y}=\left[\begin{array}{ll}\mathbf{y}_{1} & \mathbf{y}_{2}\end{array}\right]^{T}$ contains the bit representation of the two 8PSK symbols at the output of the encoder in its rows. The columns of this matrix are viewed as block codes of length equal to 2 bits. Hence, the same matrix can be represented as $\mathbf{y}=\left[\begin{array}{lll}\mathbf{y}^{2} & \mathbf{y}^{1} & \mathbf{y}^{0}\end{array}\right]$. Define the code $\mathbf{C}_{m}$ that contains $2^{3-m}$ codewords, where $m=1,2,3$ as the code to be chosen as a column of the output matrix. The first code $\mathbf{C}_{0}$ is a $(2,2)$ block code with codewords $[0,0]^{T},[0,1]^{T},[1,0]^{T}$ and $[1,1]^{T}$ and $\mathrm{HD}=1$. The second code $\mathbf{C}_{1}$ is a $(2,1)$ code with codewords $[0,0]^{T},[1,1]^{T}$ and $\mathrm{HD}=2$. The last code $\mathbf{C}_{2}$ is a $(2,0)$ block code with codeword $[0,0]^{T}$ and $H D=\infty$. The partitioning is performed such that the symbol-wise HD is increased and the SSED is increased if the former cannot be increased.

The set at partitioning level $0, \Omega^{0}$, contains all possible signal points and it can be described as $\Omega^{0}=\Omega\left(\mathbf{C}_{0}, \mathbf{C}_{0}, \mathbf{C}_{0}\right)$. So, by substituting the bit representation of each of the three codes $\mathbf{C}_{0}$ in the output matrix, all signal points in the set are generated. The set at the next partitioning level contains half the number of signal points in $\Omega^{0}$. The choice of the code to be partitioned among the three codes $\mathbf{C}_{0}$ determines the resulting set partitioning. Since there are eight branches departing from each state in the trellis required to be constructed, it is needed to have eight signal points in each subset, and hence the third partitioning level will be enough for this case. For fading channels, subsets of the 3rd partitioning level are chosen so that to maximize the symbol-wise $\mathrm{HD}$ of the generated subsets, which is $\Omega^{3}=\Omega\left(\mathbf{C}_{1}, \mathbf{C}_{1}, \mathbf{C}_{1}\right)$. This yields eight signal points in the first subset. In order to find the other subsets, the generated subset from $\Omega^{3}$ is referred to as $\Omega^{3}(0)$. The other subsets, $\Omega^{3}(1)$ to $\Omega^{3}(7)$, are generated using the generator vectors $\left(t^{p}\right)$. They are defined for this case as $\left(t^{0}\right)^{T}=\left[\begin{array}{ll}0 & 1\end{array}\right],\left(t^{1}\right)^{T}=\left[\begin{array}{ll}0 & 2\end{array}\right]$, and $\left(t^{2}\right)^{T}=\left[\begin{array}{ll}0 & 4\end{array}\right]$. The other subsets use these vectors such that

$$
\Omega^{p}(z)=\Omega^{p}(0)+\sum_{j=0}^{p-1} z^{j} t^{j} \quad(\bmod 8),
$$

where, $z=\sum_{j=0}^{p-1} 2^{j} z^{j}$ and $z^{j}$ is the $j$ th bit constituting the integer $z$.

The naturally mapped 8PSK signal constellation is shown in Figure 2, where the set partitioning of the 4D 8PSK signal space used in the first design approach is shown in Figure 3a. Also, the trellis diagram of the 8PSK2 code is shown in Figure $3 \mathrm{~b}$. From the trellis, signals from the same subset are chosen as labels of branches leaving the same encoder's state. Similarly, labels of branches entering to the same state are set to have the maximum HD. The ST minimum time diversity and minimum squared product distance of the code are 2 and 3.03, respectively. Also, the multiplicity of the shortest error event is one. When the asymptotic coding gain of this code

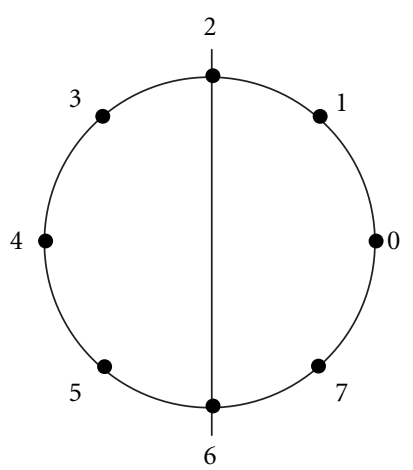

FIGURE 2: Naturally mapped 8PSK signal constellation.

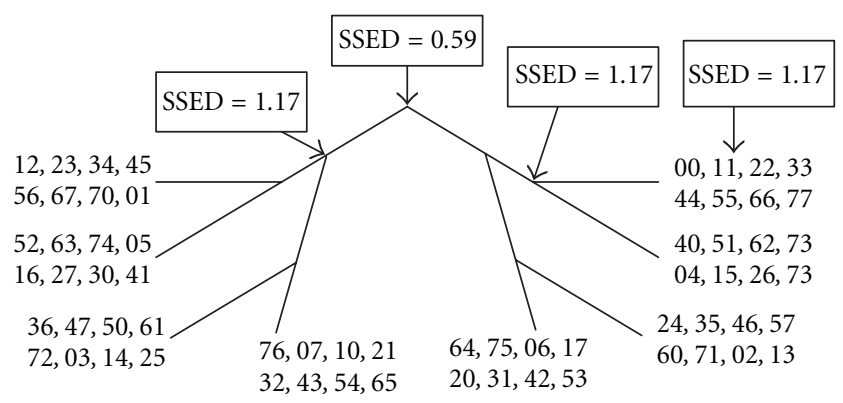

(a)

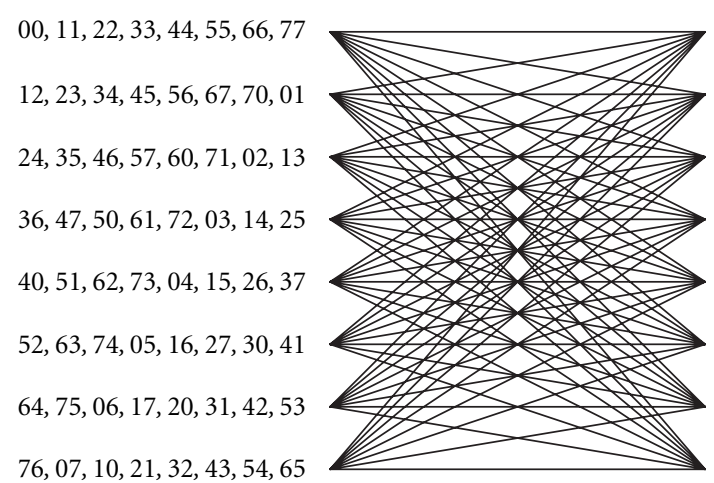

(b)

Figure 3: Trellis diagrams of 8PSK2 (8-state) $3 \mathrm{bits} / \mathrm{s} / \mathrm{Hz}$.

over the 8PSK1 code is computed, the 8PSK2 code shows a coding gain of $1.65 \mathrm{~dB}$.

The second design approach maximizes the SSED between ST symbols at branches leaving from or entering to the same state. Then, the branch-wise HD is maximized. These two parameters are equivalent to the ST minimum time diversity and minimum squared product distance of the ST code, respectively. The code designed using this approach is referred to as the 8PSK3. This approach satisfies the design criteria of ST codes in [4] since it maximizes the design parameters directly. In this approach, the $4 \mathrm{D} 8 \mathrm{PSK}$ signal space is partitioned so that the SSED between sig- 


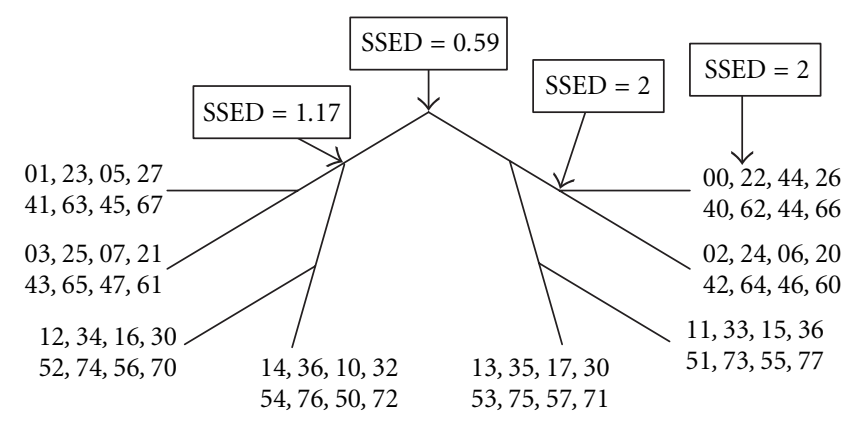

(a)

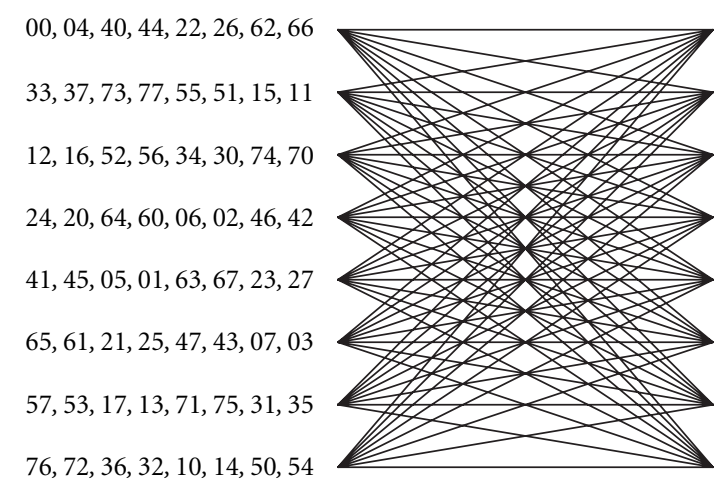

(b)

FIgURE 4: Trellis diagrams of 8PSK2 (8-state) $3 \mathrm{bits} / \mathrm{s} / \mathrm{Hz}$.

nals in each pair increases each time the partitioning is performed. Since maximizing the SSED of the code is the design criterion of trellis codes over AWGN channels, then the set partitioning optimized for AWGN channels can be utilized. The set partitioning for multidimensional MPSK signal space for AWGN channels was presented in [16]. The same terminologies used for the first approach in defining set partitioning are used here. In this case, the subset at partitioning level $3, \Omega^{3}$ is different. It is defined as $\Omega^{3}=\Omega\left(\mathbf{C}_{0}, \mathbf{C}_{1}, \mathbf{C}_{2}\right)$, where the generator vectors are $\left(t^{0}\right)^{T}=\left[\begin{array}{ll}0 & 1\end{array}\right],\left(t^{1}\right)^{T}=\left[\begin{array}{ll}1 & 1\end{array}\right]$, and $\left(t^{2}\right)^{T}=\left[\begin{array}{ll}0 & 2\end{array}\right]$.

The set partitioning of the 4D 8PSK signal space using the second approach is shown in Figure 4a. Also, the trellis diagram of the 8PSK3 code is shown in Figure 4b. In the design process, signal labels of branches leaving from the same state are drawn from the same subset. Also, signal labels of branches entering to the same state have the SSED maximized. By following these rules, it is insured that the designed code gives the maximum possible ST minimum time diversity and minimum squared product distance. For this code, the two parameters are 2 and 10.34, respectively. The shortest error event occurs with a multiplicity of one, yielding an asymptotic coding gain of $4.3 \mathrm{~dB}$ over the $8 \mathrm{PSK} 1$ scheme. It is clear that the ST minimum squared product distance is much larger than those of both 8PSK1 and 8PSK2 codes, causing significant performance improvement.

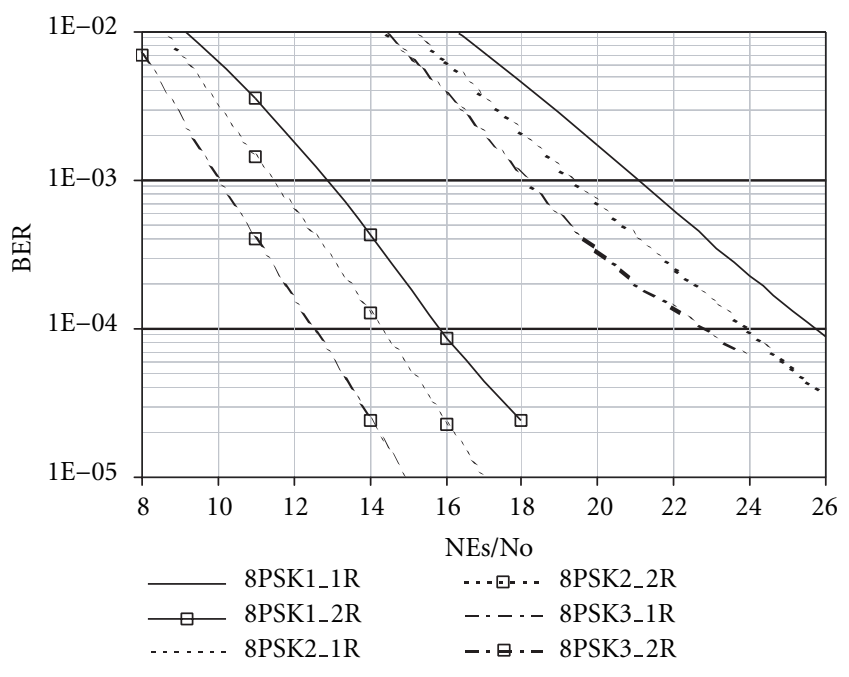

FIgure 5: Performance of the 8PSK codes for $1 \mathrm{R}$ and $2 \mathrm{R}$ antenna over ideally interleaved fading channels.

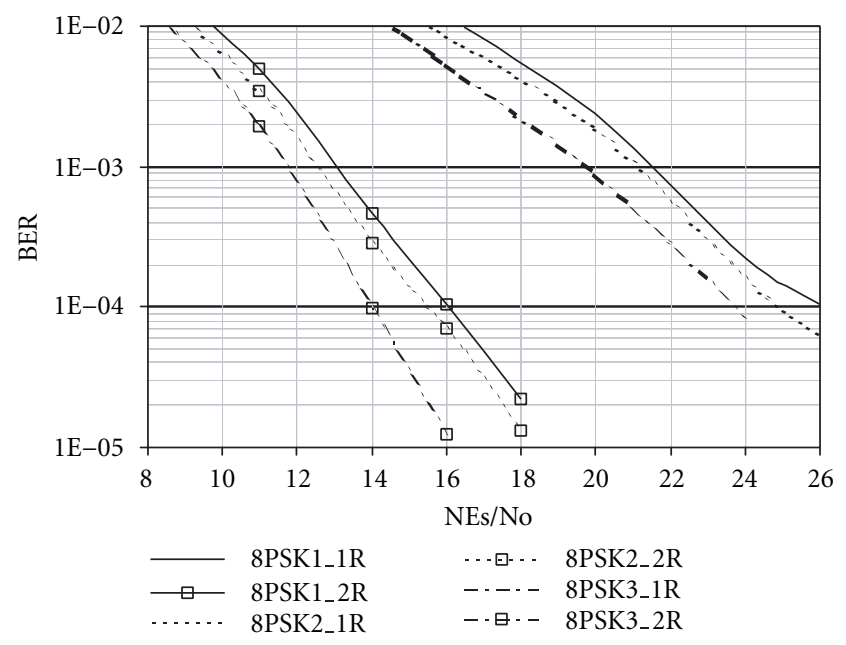

Figure 6: Performance of the 8PSK codes for $1 \mathrm{R}$ and $2 \mathrm{R}$ antenna over correlated fading channels with $f_{D} T=0.005$ and a $25 \times 16$ vector block interleaver.

\section{PERFORMANCE COMPARISONS}

The two proposed coding schemes (8PSK2 and 8PSK3) are compared to the 8PSK1 code designed in [4]. They are tested under time-varying fading environments. First, ideal interleaving that yields independent fading channels is assumed. It is presented here to show the ideal performance of the codes. Figure 5 shows the performance of the three codes for the cases of one and two receive antennas. It is clear that the 8PSK 3 code is the best followed by the 8PSK2 code. Both codes have the same ST minimum time diversity. However, the 8 PSK 3 code has a higher minimum squared product distance. The 8 PSK 3 code provides a coding gain of $4 \mathrm{~dB}$ over the 8 PSK 1 code and almost $1.3 \mathrm{~dB}$ over the 8 PSK2 code at a bit error rate (BER) of $10^{-3}$. 
It is observed that the gains of the 8PSK3 scheme over both 8PSK1 and 8PSK2 schemes are higher in the case of two receive antennas. This is because the design approach in the 8PSK3 scheme maximizes the SSED, whose contribution becomes more dominant as the channel approaches the Gaussian channel (i.e., as the number of receive antennas increases). The opposite is observed in the case of the 8PSK2 scheme, where the effect of the symbol-wise HD decreases in the case of two receive antennas because of the space diversity provided at the receiver.

Figure 6 shows the performance of the three codes over fading channels with a fading rate $\left(f_{D} T\right)$ of 0.005 . The transmitted symbols at each antenna are interleaved using a $25 \times 16$ block interleaver. This interleaver size is not enough to break the memory of the channel, and hence it is considered to be improper. This case is studied in order to test the codes under nonideal situations. The results show that the 8PSK3 code is still the best followed by 8PSK2. The gains of 8PSK3 and 8PSK2 codes over 8PSK1 code are slightly less than that for the ideally interleaved fading channel.

\section{CONCLUSIONS}

Two ST coded 8PSK schemes have been proposed. The first scheme is designed using signal space set partitioning for fading channels, and hence maximizes the symbol-wise HD. On the other hand, the second code utilizes the set partitioning for AWGN (to maximize the SSED) and then maximizes the branchwise HD. The proposed codes were simulated over ideally interleaved fading channels and also over channels with improper interleaving. Simulation results showed that these coding schemes outperform the 8PSK ST codes presented in the literature.

\section{ACKNOWLEDGMENT}

The authors wish to acknowledge the support of King Fahd University of Petroleum and Minerals provided to conduct this research.

\section{REFERENCES}

[1] B. Sklar, "Rayleigh fading channels in mobile digital communication systems-part I: characterization," IEEE Communications Magazine, vol. 35, no. 9, pp. 136-146, 1997.

[2] J. Poakis, Digital Communications, McGraw-Hill, New York, NY, USA, 1989.

[3] A. Wittneben, "A new bandwidth efficient transmit antenna modulation diversity scheme for linear digital modulation," in Proc. IEEE International Conference on Communications, pp. 1630-1634, Geneva, 1993.

[4] V. Tarokh, N. Seshadri, and A. R. Calderbank, "Space-time codes for high data rate wireless communication: performance criterion and code construction," IEEE Transactions on Information Theory, vol. 44, no. 2, pp. 744-765, 1998.

[5] S. Alamouti, "A simple transmit diversity technique for wireless communications," IEEE Journal on Selected Areas in Communications, vol. 16, no. 8, pp. 1451-1458, 1998.

[6] V. Tarokh, S. Alamouti, and P. Poon, "New detection schemes for transmit diversity with no channel estimation," in Proc.
IEEE 1998 International Conference on Universal Personal Communications, pp. 917-920, October 1998.

[7] S. Alamouti, V. Tarokh, and P. Poon, "Trellis-coded modulation and transmit diversity: Design criteria and performance evaluation," in Proc. IEEE 1998 International Conference on Universal Personal Communications, pp. 703-707, October 1998.

[8] S. Baro and A. Hansmann, "Improved codes for space-time trellis-coded modulation," IEEE Communications Letters, vol. 4, no. 1, pp. 20-22, 2000.

[9] Q. Yan and R. Blum, "Optimum space-time convolutional codes," in Proc. IEEE 2000 Wireless Communications and Networking Conference, pp. 1351-1355, September 2000.

[10] G. Bauch, "Concatenation of space-time block codes and "turbo"-TCM," in Proc. IEEE International Conference on Communications, vol. 2, pp. 1202-1206, Vancouver, British Columbia, Canada, June 2000.

[11] Y. Liu, M. P. Fitz, and O. Y. Takeshita, "Full rate space-time turbo codes," IEEE Journal on Selected Areas in Communications, vol. 19, no. 5, pp. 969-980, 2001.

[12] S. Zummo and S. Al-Semari, "Design of space-time QPSK codes for Rayleigh fading channel," in Proc. 11th IEEE International Symposium on Personal, Indoor, and Mobile Radio Communications, pp. 504-508, September 2000.

[13] S. Zummo and S. Al-Semari, "Design of 16-QAM space-time codes for rapid Rayleigh fading channels," in Proc. 10th annual MPRG Symposium on Wireless Personal Communications, June 2000.

[14] S. H. Jamali and T. Le-Ngoc, Coded-Modulation Techniques for Fading Channels, Kluwer Academic, Boston, Mass, USA, 1994.

[15] E. Leonardo, L. Zhang, and B. Vucetic, "Multidimensional M-PSK trellis codes for fading channels," IEEE Transactions on Information Theory, vol. 42, no. 4, pp. 1093-1108, 1996.

[16] S. Pietrobon, R. Deng, A. Lafanechere, G. Ungerboeck, and D. Costello, "Trellis coded multidimensional phase modulation," IEEE Transactions on Information Theory, vol. 36, no. 1, pp. 63-89, 1990.

Salam A. Zummo was born in 1976 in Saudi Arabia. He received his B.S. and M.S. degrees in electrical engineering from King Fahd University of Petroleum and Minerals (KFUPM), Dhahran, Saudi Arabia, in 1998 and 1999, respectively. In 2000, he joined the Electrical Engineering and Computer Science Department at the University of Michigan, Ann Arbor, where he is currently a Ph.D. candidate. His main research interests are in space-time codes, error control coding, channel estimation and iterative receivers for wireless systems.

Saud A. Al-Semari received his B.S. and M.S. degrees in electrical engineering from KFUPM in 1991 and 1992, respectively. He received his Ph.D. degree from the University of Maryland at College Park, USA, in December 1995. He is currently an Associate Professor of Electrical Engineering at KFUPM. He is also the Director of the Information Technology Center at KFUPM. Dr. Al-Semari pursues research in a range of topics related to wireless communication systems including error control coding, diversity, fading, multiple-access and security. 